Д.В. Кулеш ${ }^{1}$, С.И. Колесников ${ }^{1}$, В.В. Долгих ${ }^{1}$, С.В. Шойко ${ }^{2}$, Н.Н. Абашин ${ }^{1}$, Л.Н. Лебедева ${ }^{1}$

${ }^{1}$ Научный центр проблем здоровья семьи и репродукции человека Сибирского отделения РАМН, Иркутск, Российская Федерация

2 Территориальный фонд обязательного медицинского страхования граждан Иркутской области, Иркутск, Российская Федерация

\title{
Медико-эпидемиологические
} и методологические подходы к прогнозированию показателей, характеризующих здоровье подростков на современном этапе

\begin{abstract}
Данные об особенностях формирования здоровья разных групп населения в процессе развития страны и ее отдельных регионов являются важной информационной основой для разработки и осуществления мер по сохранению и укреплению общественного здоровья. Целью исследования явилось определение и прогнозирование тендениии изменения показателей первичной заболеваемости и распространенности болезней у подросткового населения на ближайшие 10 лет. Материалами для анализа послужили данные сводных годовых отчетных форм № 12 «Сведения о числе заболеваний, зарегистрированных у больных, проживающих в районе обслуживания лечебного учреждения» по региону в динамике за 10-летний период (2001-2010). Осуществлялся расчет и анализ динамики интегральных коэффициентов, определялся уровень нарушения состояния здоровья подростков от низкого до высокого. Темпы прироста показателей частоты заболеваемости и распространенности болезней оценивали путем расчета показателей динамического ряда с определением коэффициента аппроксимации $R^{2}$. B статье представлены результаты прогнозирования на ближайшие 10 лет у подросткового контингента населения (15-17лет) изменений показателей первичной заболеваемости и распространенности болезней. Для этого была применена разработанная авторами методика. С учетом обнаруженных тенденций установлен прогнозируемый прирост данных показателей на 40,7-50,2\%. По полу прогнозируемая динамика показателей предполагает их прирост у юношей на 42,1-58,9\%, у девушек - на 31,2-49,6\%. Эти данные негативно характеризуют прогнозируемую на ближайшее время региональную тенденцию динамики показателей заболеваемости у анализируемого контингента населения. Они должны быть учтены при решении вопросов сохранения и укрепления здоровья подрастающего поколения, в т.ч. при формировании региональных соииальных программ и программ здравоохранения.
\end{abstract}

Ключевые слова: подростки, первичная заболеваемость, распространенность, прогнозирование.

D.V. Kulesh', S.I. Kolesnikov', V.V. Dolgich¹, S.V. Shoyko², N.N. Abashin², L.N. Lebedeva1

${ }^{1}$ Scientific Centre of the Family Health and Human Reproduction Problems, Siberian Branch of Russian Academy of Medical Sciences, Irkutsk, Russian Federation

2 Irkutsk Regional Fund of Obligatory Medical Insurance, Irkutsk, Russian Federation

\section{Medical-Epidemiological and Methodological Approaches to Forecasting Indicators of Adolescent Health in the Modern Period}

Data on features of formation of health of different groups of the population in the development process of the country and its regions are an important information base for the development and implementation of measures for the maintenance and strengthening of public health. The aim of the study was to determine and predict trends in indicators of primary disease incidence and prevalence among adolescent population over the next 10 years. Materials provided to analyze the data of the consolidated annual report forms № 12 "The number of registered diseases in patients living in the service area of the medical establishment" for showing trends over the 10-year period (2001-2010). Calculation and analysis of dynamics were integral coefficients, determined the level of adolescent health violations from "low" to "high". Incidence rate of incidence and prevalence of disease were evaluated by calculating the dynamic coefficient of determination $R$ approximation. The article presents results of forecasting over the next 10 years in the adolescent population (15-17 years) changes the primary indicators incidence and prevalence of diseases. This technique was used by the authors. In view of the projected increase in detected trends data set of indicators at 40,7-50,2\%. Sex predicted dynamics of increase in juniors at 42,1-58.9\%, the girls - the 31,2-49,6\%. These data characterize negatively predicted for the near future a regional trend in incidence rates in the analyzed populations. They must be taken into account when deciding on the preservation and strengthening of health of the rising generation, including - in shaping regional social programs and health care programs.

Key words: adolescents, primary incidence, prevalence, prediction. 


\section{Введение}

Состояние здоровья подрастающего поколения важный показатель благополучия общества и государства, отражающий не только настоящую ситуацию, но и прогноз на будущее. Руководители России неоднократно подчеркивали приоритетность развития детского здравоохранения. Это определяется тем, что здоровье детского населения является не только интегральным показателем его качества [1-4], но и составляет фундаментальную основу для формирования потенциала здоровья взрослых членов общества. Именно поэтому в настоящее время общепризнанно, что задача охраны здоровья детей и подростков на современном этапе развития общества относится к числу первостепенных. В ближайшие годы общество может столкнуться с серьезными проблемами здоровья и социализации молодежи [5]. В целях совершенствования системы охраны здоровья граждан, в т.ч. и детского населения, была разработана Государственная программа «Развитие здравоохранения в Российской Федерации до 2020 г.» [6]. В связи с вышесказанным большую значимость приобретают научные исследования по изучению особенностей состояния здоровья подростков, в т.ч. с позиции прогнозирования региональных показателей, характеризующих заболеваемость данного контингента населения на современном этапе.

Негативная динамика показателей заболеваемости подростков старшей возрастной группы (15-17 лет) в России (в частности, в Иркутской обл.) послужила основанием для проведения настоящего исследования.

Цель исследования: определить тенденции изменения показателей первичной заболеваемости и распространенности болезней у подросткового населения на ближайшие 10 лет, что в итоге позволит прогнозировать затраты здравоохранения на охрану здоровья детей и подростков, коечный фонд и подготовку кадров.

\section{Материалы и методы}

\section{Участники исследования}

Проведен анализ показателей (по данным обращаемости) первичной заболеваемости и распространенности болезней у подросткового контингента населения (15-17 лет), проживающего в Иркутской обл. Иркутская обл. - один из ключевых регионов, заболеваемость в котором можно экстраполировать на показатели огромного по площади Восточно-Сибирского региона России с населением около 10 млн человек.

\section{Методы исследования}

Материалами для анализа послужили данные сводных годовых отчетных форм № 12 «Сведения о числе заболеваний, зарегистрированных у больных, проживающих в районе обслуживания лечебного учреждения» по региону в динамике за 10-летний период (2001-2010). Расчет и анализ динамики интегральных коэффициентов осуществлялся по методике Е.Н. Беляева, А.И. Мелехина, М.Ф. Глазкова и соавт. [7]. С помощью данной методики определяли уровень нарушения состояния здоровья подростков (от низкого до высокого). Темпы прироста показателей частоты заболеваемости и распространенности болезней оценивали с применением расчета показателей динамического ряда, его выравнивания методом наи- меньших квадратов $(\mathrm{y}=\mathrm{a}+\mathrm{bx})$ с определением коэффициента аппроксимации $\mathrm{R}^{2}$.

Прогностический уровень показателей первичной заболеваемости и распространенности болезней по соответствующим классам заболеваний рассчитан с применением разработанной нами методики прогнозирования показателей заболеваемости с расчетом и анализом показателей динамического ряда. Прогнозируемый показатель определяли по следующей формуле:

$$
\Pi=(\text { Б } \times \text { B })+A \text {, }
$$

где П - прогнозируемый показатель; Б - темп прироста показателя на конец исследуемого периода; В - абсолютное значение $1 \%$ прироста показателя на конец исследуемого периода; А - показатель на конец исследуемого периода.

\section{Статистическая обработка данных}

В исследовании использовали вычислительные процедуры методов математической статистики, реализованные в лицензионном интегрированном статистическом пакете комплексной обработки данных «STATISTICA 6.1» (StatSoft Inc., CШA) [8]. Проводился расчет интенсивных и экстенсивных показателей, оценка значимости различий. Темпы прироста показателей частоты заболеваемости оценивались по уровню коэффициента аппроксимации $-\mathrm{R}^{2}$, значение которого может быть от 0 до 1. Чем ближе он к 1, тем выше степень приближения расчетной функции к реальной ситуации.

\section{Результаты}

Интегральная оценка показала, что динамика уровня нарушений у подростков в исследуемом периоде характеризовалась интенсивным ростом. Значения интегрального показателя с учетом первичной заболеваемости соответствовали низкому уровню в 2001 г., а с учетом распространенности болезней - в период 2001-2002 гг.; умеренному - соответственно, в 20022004 и 2003-2004 гг.; повышенному - в 2005-2008 и 2005 гг. В период 2006-2008 гг. показатель нарушений с учетом впервые выявленной заболеваемости у подростков находился в зоне повышенного, а за последние 2 года (2009-2010) - в зоне высокого уровня. С учетом распространенности болезней у исследуемого контингента населения за последние 5 лет (2006-2010) уровень нарушений соответствовал высокому, демонстрируя самую негативную динамику (рис. 1).

Таким образом, интегральная оценка нарушений здоровья у подростков и динамика соответствующих интегральных коэффициентов говорят об интенсивных процессах в ухудшении здоровья подросткового населения. Особенно это характерно для периода последних 5 лет (2006-2010), за которые показатель накопленной заболеваемости (распространенности болезней) продемонстрировал наиболее негативную тенденцию и самый высокий уровень нарушений.

Относительный прирост показателей первичной заболеваемости подростков за исследуемый период в Иркутской обл. был статистически значимым $\left(\mathrm{R}^{2}=0,816\right)$ и составил $39,4 \%$ (рис. 2). Аналогичная тенденция зафиксирована как в целом по России, так и по Сибирскому федеральному округу: прирост соответствующих коэффициентов за анализируемый период составил 45,6 и $29,2 \%$, соответственно. 
Немаловажное значение в характеристике здоровья подростков имеет общая накопленная заболеваемость, или, как принято ее называть, распространенность болезней. Показатели распространенности характеризуют общее число болезней, зарегистрированных у подросткового населения не только впервые на конкретный период, но и выявленные ранее, включая хронические формы, а также преморбидные состояния, функциональные отклонения, в основном характеризующие состояния предболезни. Исследование динамики показателей распространенности болезней у анализируемого контингента населения за период 2001-2010 гг. демонстрирует статистически значимый прирост как по России в целом, так и в Сибирском федеральном округе в частности $(42,2 \%$; $\mathrm{R}^{2}=0,963$ и $27,0 \% ; \mathrm{R}^{2}=0,809$, соответственно) (рис. 3 ).

Темп прироста показателей распространенности болезней у данного контингента населения в Иркутской обл. за исследуемый период составил $37,9 \%$ (2165,4 на 1000 подростков в возрасте 15-17 лет). При этом данные показатели по региону на конец исследуемого периода оказались на 5,6\% выше, чем в Сибирском федеральном округе, и на 4,8\% ниже, чем в целом по России.

Таким образом, у подростков в возрасте 15-17 лет, проживающих в Иркутской обл., установлены тенденции прироста показателей первичной заболеваемости и распространенности болезней. Так, за последние 10 лет для изучаемого контингента населения показан наиболее значимый прирост по следующим группам показателей:

- распространенность болезней костно-мышечной системы и соединительной ткани (на 91,7\%; $\left.\mathrm{R}^{2}=0,957\right)$;

- распространенность болезней системы кровообращения (на $81,7 \%$; $\mathrm{R}^{2}=0,908$ );

- распространенность болезней нервной системы (на $83,9 \% ; \mathrm{R}^{2}=0,845$ );

- впервые выявленные болезни нервной системы (на $76,1 \% ; \mathrm{R}^{2}=0,752$ ).

Анализ показал, что в структуре первичной заболеваемости у подростков Иркутской обл. превалирует 3 основных класса патологии. На первом ранговом месте находятся болезни органов дыхания $(45,9 \%)$, на втором травмы и отравления $(7,5 \%)$, на третьем - болезни органов пищеварения $(6,1 \%)$, т.е. в структуре первичной заболеваемости подростков на долю данных классов приходится $59,5 \%$ болезней. Однако в исследовании установлено, что с наибольшим темпом увеличиваются показатели по классам, которые не всегда занимают превалирующие ранговые позиции (табл. 1).

Изучение показателей динамического ряда позволило установить, что наибольший темп прироста показателей первичной заболеваемости у подростков характерен для классов болезней, представленных в табл. 1. Из структуры преобладающих в данном случае представлены только болезни органов пищеварения (XI класс), тогда как болезни костно-мышечной системы и соединительной ткани (XIII класс) и болезни нервной системы (VI класс) не являются преобладающими в структуре первичной заболеваемости подростков, однако темп прироста по данным классам является самым высоким.

Эти данные свидетельствуют о возможном изменении структуры первичной заболеваемости подростков в перспективе, поскольку интенсивный прирост классов болезней, не являющихся преобладающими в настоящее время, может в дальнейшем вывести их на превалирующие позиции.

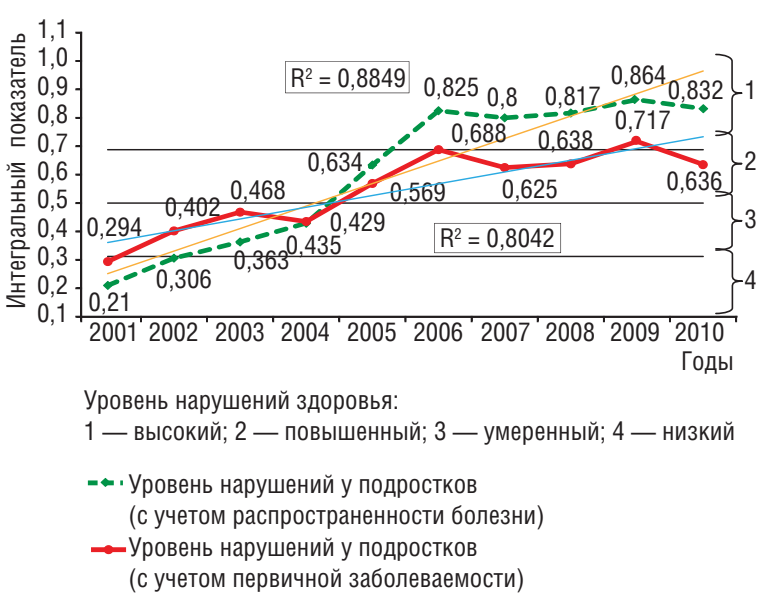

Рис. 1. Динамика интегрального показателя уровней нарушений с учетом данных о впервые выявленной заболеваемости и распространенности болезней у подростков 15-17 лет в Иркутской области за период 2001-2010 гг.

Примечание. $\mathrm{R}^{2}$ - коэффициент аппроксимации.

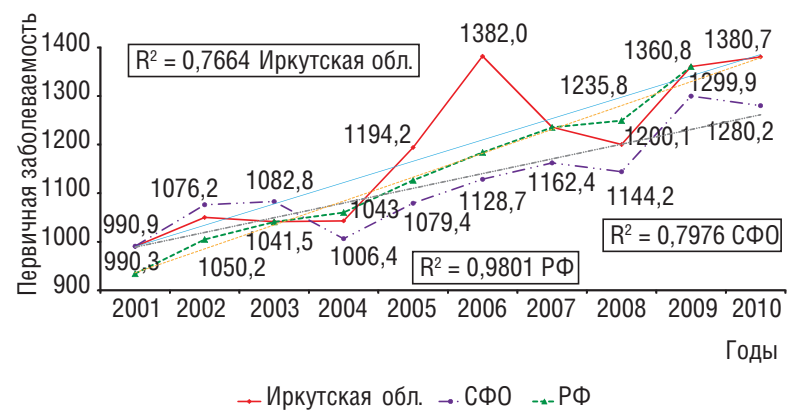

Рис. 2. Динамика показателей первичной заболеваемости подросткового населения в возрасте 15-17 лет по данным обращаемости за медицинской помощью в Российской Федерации, Иркутской области и Сибирском федеральном округе (на 1000 подростков).

Примечание (здесь и для рис. 3). СФО - Сибирский федеральный округ, РФ - Российская Федерация, $\mathrm{R}^{2}$ - коэффициент аппроксимации.

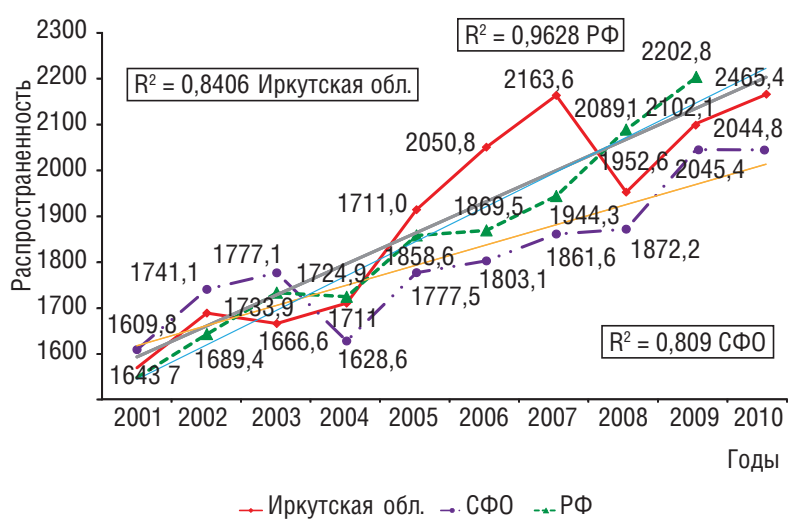

Рис. 3. Динамика показателей распространенности заболеваний среди подростков в возрасте 15-17 лет по данным обращаемости за медицинской помощью в Российской Федерации, Иркутской области и Сибирском федеральном округе (на 1000 подростков). 
По разработанной нами методике с применением показателей динамического ряда был рассчитан прогнозный уровень показателей первичной заболеваемости и распространенности болезней по соответствующим классам заболеваний на ближайшие 10 лет. Суть методики заключается в том, что для расчета прогнозируемого показателя заболеваемости учитываются фактические его значения на начало и на конец исследуемого периода с расчетом показателей динамического ряда, после чего определяется прогнозируемый показатель на ближайшую перспективу такого же числа лет, которым представлен исследуемый период. Данная методика не учитывает возможное влияние различных факторов в течение выбранного периода и предназначена только для гипотетического прогнозирования фактических анализируемых показателей при условии неизменных тенденций и их реальной динамики. Она может применяться с целью анализа текущей ситуации, определения возможных рисков и для принятия управленческих решений, влияющих на сложившиеся фактические тенденции динамики анализируемых показателей в перспективе.

Из данных, представленных в табл. 1, видно, что при условии сохраняющейся тенденции динамики показателей по классам, характеризующимся наибольшим темпом прироста, в ближайшие 10 лет впервые выявленная заболеваемость может вырасти для болезней:

- костно-мышечной системы и соединительной ткани - на $40,7 \%$;

- нервной системы - на 47,4\%;

- органов пищеварения - на $43,7 \%$.

Аналогичным образом у подростков осуществляли анализ и прогнозирование показателей распространенности болезней. Установлено, что в структуре показателя распространенности болезней у подростков Иркутской обл. преобладает патология органов дыхания $(33,3 \%)$ на втором ранговом месте - болезни органов пищеварения $(8,9 \%)$, на третьем - заболевания костно-мышечной системы и соединительной ткани $(7,6 \%)$. Они в структуре показателя распространенности составляют $1 / 2$ всех заболеваний $(49,8 \%)$.

Как и в случае с первичной заболеваемостью, не все основные классы, характеризующие структуру распространенности болезней, имеют наибольший прирост за исследуемый период (2001-2010 гг.). Из преобладающих классов в структуре показателя распространенности болезней у подростков в данном случае представлены только болезни костно-мышечной системы (XIII класс) (табл. 2). Болезни же нервной системы (VI класс) и системы кровообращения (IX класс) не являются преобладающими, однако характеризуются наибольшим темпом прироста

Эти данные, как и в случае с первичной заболеваемостью, свидетельствуют о вероятной смене в перспективе структуры показателя распространенности болезней у подростков региона.

Показано, что при сохраняющейся тенденции в ближайшие 10 лет у подростков прогнозируется прирост болезней костно-мышечной системы и соединительной ткани на 50,2\%, болезней нервной системы - на 46,2\%, болезней системы кровообращения - на $45,5 \%$.

С учетом установленных закономерностей и классов болезней с наиболее интенсивным приростом в исследуемом периоде на ближайшие 10 лет у подростков были спрогнозированы показатели впервые выявленной заболеваемости и распространенности болезней по половой принадлежности.

Прогнозируемый прирост показателей заболеваемости на ближайшие 10 лет представлен в табл. 3.

Таблица 1. Прогноз показателей первичной заболеваемости подростков в возрасте 15-17 лет в Иркутской обл. по классам болезней на ближайшие 10 лет

\begin{tabular}{|c|c|c|c|c|c|c|c|c|}
\hline \multirow{3}{*}{$\begin{array}{l}\text { № } \\
\text { П/ } / \text { II }\end{array}$} & \multirow[t]{3}{*}{ Класс МКБ-10 } & \multirow{2}{*}{\multicolumn{2}{|c|}{$\begin{array}{c}\text { Показатель } \\
\text { на } 1000 \text { подростков } \\
(15-17 \text { лет) }\end{array}$}} & \multicolumn{4}{|c|}{ Показатели динамического ряда } & \multirow{3}{*}{$\begin{array}{c}\text { Прогнозируемый } \\
\text { показатель } \\
\text { (П) к } 2020 \text { г.: } \\
\text { (графа7×графа 8) + } \\
\text { графа } 4\end{array}$} \\
\hline & & & & \multirow[t]{2}{*}{$\begin{array}{c}\text { Абс. } \\
\text { прирост }\end{array}$} & \multirow{2}{*}{$\begin{array}{c}\text { Темі } \\
\text { роста, } \\
\%\end{array}$} & \multirow{2}{*}{$\begin{array}{c}\text { Темп } \\
\text { прироста, } \\
\% \text { (Б) }\end{array}$} & \multirow{2}{*}{$\begin{array}{l}\text { Абс. значение } \\
1 \% \text { прироста } \\
\text { (В) }\end{array}$} & \\
\hline & & 2001 г. & 2010 г. (A) & & & & & \\
\hline 1 & 2 & 3 & 4 & 5 & 6 & 7 & 8 & 9 \\
\hline 1 & $\begin{array}{l}\text { Болезни костно-мышечной системы } \\
\text { и соединительной ткани (XIII) }\end{array}$ & 42,5 & 74,9 & 32,4 & 176,2 & 76,2 & 0,4 & 105,4 \\
\hline 2 & Болезни нервной системы (VI) & 27,3 & 48,1 & 20,8 & 176,1 & 76,1 & 0,3 & 70,9 \\
\hline 3 & Болезни органов пищеварения (XI) & 48,5 & 84,2 & 35,7 & 173,6 & 73,6 & 0,5 & 121,0 \\
\hline
\end{tabular}

Примечание. П - прогнозируемый показатель; Б - темп прироста показателя на конец исследуемого периода; В - абсолютное значение $1 \%$ прироста показателя на конец исследуемого периода; А - показатель на конец исследуемого периода.

Таблица 2. Прогноз показателей распространенности заболеваний подростков в возрасте $15-17$ лет в Иркутской обл. по классам болезней на ближайшие 10 лет

\begin{tabular}{|c|c|c|c|c|c|c|c|c|}
\hline \multirow{3}{*}{$\begin{array}{l}\text { № } \\
\text { ПI/II }\end{array}$} & \multirow[t]{3}{*}{ Класс МКБ-10 } & \multirow{2}{*}{\multicolumn{2}{|c|}{$\begin{array}{c}\text { Показатель } \\
\text { на } 1000 \text { подростков } \\
(15-17 \text { лет) }\end{array}$}} & \multicolumn{4}{|c|}{ Показатели динамического ряда } & \multirow{3}{*}{$\begin{array}{c}\text { Прогнозируемый } \\
\text { показатель } \\
\text { (П) к } 2020 \text { г.: } \\
\text { (графа7×ггафа 8) + } \\
\text { графа } 4\end{array}$} \\
\hline & & & & \multirow[t]{2}{*}{$\begin{array}{l}\text { Абс. } \\
\text { прирост }\end{array}$} & \multirow{2}{*}{$\begin{array}{c}\text { Темп } \\
\text { роста, } \\
\%\end{array}$} & \multirow{2}{*}{$\begin{array}{c}\text { Темп } \\
\text { прироста, } \\
\% \text { (Б) }\end{array}$} & \multirow{2}{*}{$\begin{array}{l}\text { Абс. значение } \\
1 \% \text { прироста } \\
\text { (В) }\end{array}$} & \\
\hline & & 2001 r. & 2010 г. (A) & & & & & \\
\hline 1 & 2 & 3 & 4 & 5 & 6 & 7 & 8 & 9 \\
\hline 1 & $\begin{array}{l}\text { Болезни костно-мышечной системы } \\
\text { и соединительной ткани (XIII) }\end{array}$ & 85,8 & 164,5 & 78,7 & 191,7 & 91,7 & 0,9 & 247,0 \\
\hline 2 & Болезни нервной системы (VI) & 69,1 & 127,1 & 58,0 & 183,9 & 83,9 & 0,7 & 185,8 \\
\hline 3 & Болезни системы кровообращения (IX) & 19,7 & 35,8 & 16,1 & 181,7 & 81,7 & 0,2 & 52,1 \\
\hline
\end{tabular}


Таблица 3. Прогноз показателей впервые выявленной заболеваемости и распространенности болезней у юношей в возрасте $15-17$ лет в Иркутской обл. по классам болезней на ближайшие 10 лет

\begin{tabular}{|c|c|c|c|c|c|c|c|c|}
\hline \multirow{3}{*}{$\begin{array}{l}\text { № } \\
\text { п/II }\end{array}$} & \multirow[t]{3}{*}{ Класс МКБ-10 } & \multirow{2}{*}{\multicolumn{2}{|c|}{$\begin{array}{c}\text { Показатель на } \\
1000 \text { подростков } \\
\text { (15-17 лет) }\end{array}$}} & \multicolumn{4}{|c|}{ Показатели динамического ряда } & \multirow{3}{*}{$\begin{array}{c}\text { Прогнозируемый } \\
\text { показатель } \\
\text { (П) к } 2020 \text { г.: } \\
\text { (графа7×графа 8) + } \\
\text { графа } 4\end{array}$} \\
\hline & & & & \multirow[t]{2}{*}{$\begin{array}{l}\text { Абс. } \\
\text { прирост }\end{array}$} & \multirow{2}{*}{$\begin{array}{c}\text { Темп } \\
\text { роста, } \\
\%\end{array}$} & \multirow{2}{*}{$\begin{array}{c}\text { Темп } \\
\text { прироста, } \\
\% \text { (Б) }\end{array}$} & \multirow{2}{*}{$\begin{array}{l}\text { Абс. значение } \\
1 \% \text { прироста } \\
\text { (В) }\end{array}$} & \\
\hline & & 2001 г. & 2010 г. (A) & & & & & \\
\hline 1 & 2 & 3 & 4 & 5 & 6 & 7 & 8 & 9 \\
\hline \multicolumn{9}{|c|}{ Впервые выявленная заболеваемость } \\
\hline 1 & $\begin{array}{l}\text { Болезни костно-мышечной системы } \\
\text { и соединительной ткани (XIII) }\end{array}$ & 41,5 & 84,5 & 43,0 & 203,6 & 103,6 & 0,4 & 125,9 \\
\hline 2 & Болезни органов пищеварения (XI) & 43,4 & 85,9 & 42,5 & 197,9 & 97,9 & 0,4 & 125,1 \\
\hline 3 & Болезни системы кровообращения (IX) & 11,5 & 22,3 & 10,8 & 193,9 & 93,9 & 0,1 & 31,7 \\
\hline \multicolumn{9}{|c|}{ Распространенность болезней } \\
\hline 1 & $\begin{array}{l}\text { Болезни костно-мышечной системы } \\
\text { и соединительной ткани (XIII) }\end{array}$ & 78,7 & 187,4 & 108,7 & 238,1 & 138,1 & 0,8 & 297,9 \\
\hline 2 & Болезни органов пищеварения (XI) & 104,4 & 186,1 & 81,7 & 178,3 & 78,3 & 1,0 & 264,4 \\
\hline 3 & Болезни системы кровообращения (IX) & 22,1 & 48,7 & 26,6 & 220,4 & 120,4 & 0,2 & 72,8 \\
\hline
\end{tabular}

Таблица 4. Прогноз показателей впервые выявленной заболеваемости и распространенности болезней у девушек в возрасте $15-17$ лет в Иркутской обл. по классам болезней на ближайшие 10 лет

\begin{tabular}{|c|c|c|c|c|c|c|c|c|}
\hline \multirow{3}{*}{$\begin{array}{l}\text { № } \\
\text { ПI/ா }\end{array}$} & \multirow[t]{3}{*}{ Класс МКБ-10 } & \multirow{2}{*}{\multicolumn{2}{|c|}{$\begin{array}{c}\text { Показатель на } \\
1000 \text { подростков } \\
\text { (15-17 лет) }\end{array}$}} & \multicolumn{4}{|c|}{ Показатели динамического ряда } & \multirow{3}{*}{$\begin{array}{c}\text { Прогнозируемый } \\
\text { показатель } \\
\text { (П) к } 2020 \text { г.: } \\
\text { (графа7×графа 8) + } \\
\text { графа } 4\end{array}$} \\
\hline & & & & \multirow[t]{2}{*}{$\begin{array}{c}\text { Абс. } \\
\text { прирост }\end{array}$} & \multirow{2}{*}{$\begin{array}{c}\text { Темп } \\
\text { роста, } \\
\%\end{array}$} & \multirow{2}{*}{$\begin{array}{c}\text { Темп } \\
\text { прироста, } \\
\% \text { (Б) }\end{array}$} & \multirow{2}{*}{$\begin{array}{l}\text { Абс. значение } \\
1 \% \text { прироста } \\
\text { (В) }\end{array}$} & \\
\hline & & 2001 г. & 2010 г. (A) & & & & & \\
\hline 1 & 2 & 3 & 4 & 5 & 6 & 7 & 8 & 9 \\
\hline \multicolumn{9}{|c|}{ Впервые выявленная заболеваемость } \\
\hline 1 & Болезни нервной системы (VI) & 30,8 & 58,6 & 27,8 & 190,3 & 90,3 & 0,3 & 85,7 \\
\hline 2 & Болезни органов пищеварения (XI) & 53,8 & 82,5 & 28,7 & 153,4 & 53,3 & 0,5 & 109,2 \\
\hline 3 & Болезни органов дыхания (X) & 450,7 & 682,9 & 232,2 & 151,5 & 51,5 & 4,5 & 914,7 \\
\hline \multicolumn{9}{|c|}{ Распространенность болезней } \\
\hline 1 & Болезни нервной системы (VI) & 76,2 & 144,5 & 68,3 & 189,6 & 89,6 & 0,8 & 216,2 \\
\hline 2 & $\begin{array}{l}\text { Болезни костно-мышечной системы } \\
\text { и соединительной ткани (XIII) }\end{array}$ & 93,1 & 140,6 & 47,5 & 151,0 & 51,0 & 0,9 & 186,5 \\
\hline 3 & Болезни органов пищеварения (XI) & 134,9 & 199,5 & 64,6 & 147,9 & 47,9 & 1,3 & 261,8 \\
\hline
\end{tabular}

У юношей показатели первичной заболеваемости могут увеличиться по классу болезней костно-мышечной системы и соединительной ткани на 48,9\%, по классу болезней органов пищеварения - на 45,6\%, по классу болезней системы кровообращения - на 49,5\%. При этом в этой же группе накопленная заболеваемость (распространенность болезней) за тот же период вырастет по классу болезней костно-мышечной системы и соединительной ткани на 58,9\%, по классу болезней органов пищеварения - на 42,1\%, по классу болезней системы кровообращения - на 49,5\%.

Аналогичным образом был спрогнозирован прирост соответствующих показателей у девушек (табл. 4). Первичная заболеваемость у них в ближайшие 10 лет может увеличиться по классу болезней нервной системы на 46,2\%, по классу болезней органов пищеварения - на $32,4 \%$, по классу болезней органов дыхания - на 33,9\%. Прирост распространенности болезней у девушек в ближайшие 10 лет составит по классу болезней нервной системы 49,6\%, по классу болезней костно-мышечной системы и соединительной ткани - 32,6\%, по классу болезней органов пищеварения $-31,2 \%$.

\section{Заключение}

Установлено, что наибольший прирост показателей первичной заболеваемости и распространенности болезней у подростков характеризуется классами болезней, не всегда определяющими на текущий момент их основную структуру. Этим в ближайшей перспективе может быть обусловлено изменение структуры как первичной заболеваемости, так и распространенности болезней. С учетом обнаруженных региональных тенденций прогнозируемый рост данных показателей в ближайшие 10 лет в целом у подростков составит 40,7-50,2\%. По половой принадлежности динамика данных коэффициентов предполагает прирост у юношей на 42,1-58,9\%, у девушек - на $31,2-49,6 \%$.

Данная негативная тенденция предполагает необходимость использования прогнозирования при разработке региональных социальных программ, в т.ч. программ здравоохранения. Постановка и программное решение реальных задач с учетом прогнозируемых тенденций обеспечат ведение эффективной управленческой политики, направленной на результат и в конечном счете на достижение цели укрепления и сохранения здоровья подрастающего поколения. 


\section{REFERENCES}

1. Antonova E.V. Zdorov'e rossiiskikh podrostkov 15-17 let: sostoyanie, tendentsii i nauchnoe obosnovanie programmy ego sokhraneniya i ukrepleniya. Avtoref. dis. ... dokt. med. nauk [Health of Russian teenagers aged 15-17 years old: Status, Trends and scientific rationale of the program, health preservation and strengthening. Abstract of Dissertation for the degree of Doctor of medical science]. Moscow. 2011. 24 p.

2. Dolgikh V.V., Kulesh D.V. The state of children health and adolescents living in the Irkutsk region. Voprosy Sovremennoi Pediatrii - Current Pediatrics. 2006; 1 (5): 180-181.

3. Gaidarov G.M., Dolgikh V.V., Kulesh D.V., Antonova E.V., Shkandylo T.P. Medical and social and organizational aspects of teenagers health protection in modern conditions. Sibirskii meditsinskii zhurnal (Irkutsk) - Siberian Medical Journal (Irkutsk). 2007; 3 (70): 5-9.

4. Polunina N.V., Popov V.V. Differentiate approach to formation of indicators for younger generation's health. Glavvrach - Medical director. 2006; 11: 49-52.
5. Lisitsyn Yu.P. Obshchestvennoe zdorov'e i zdravookhranenie: uchebnik [Public health and health care: a textbook.]. Moscow: GEOTAR-Media. 2010. 512 p.

6. Baranov A.A. Il'in A.G. The main trends of children's health dynamics in the Russian Federation. Ways to solve problems. Vestnik Rossiiskoi akademii meditsinskikh nauk - Annals of the Russian Academy of Medical Sciences. 2011; 6: 12-18.

7. Belyaev E.N., Melekhin A.I., Glazkova M.F., Ponomareva O.V., Zaitseva N.V., Shur P.Z., Gimervert L.A. Integral'naya otsenka sostoyaniya zdorov'ya naseleniya na territoriyakh: metodicheskie ukazaniya [Integral assessment of health population on the territories]. Moscow. 1995. 33 p.

8. Yunkerov V.I., Grigor'ev S.G. Matematiko-statisticheskie metody obrabotki dannykh meditsinskikh issledovanii [Mathematical and statistical methods of processing of medical research data]. St. Petersburg.2002. 266 p.

\section{FOR CORRESPONDENCE}

Kulesh Dmitrii Vladimirovich, PhD, Head of the Laboratory of Public Health Problems, Scientific Center of Family Health and Human Reproduction Problems Siberian Branch of Russian Academy of Medical Sciences Address: 664003, Irkutsk, Timirjazeva St., 16; tel.: (83952) 20-73-67; e-mail: iphr@sbamsr.irk.ru

Kolesnikov Sergei Ivanovich, PhD, RAMS academician, Leading Research Worker, Laboratory of Assisted Reproductive Technologies and Perinatal Medicine, Scientific Center of Family Health and Human Reproduction Problems Siberian Branch of Russian Academy of Medical Sciences

Address: 664003, Irkutsk, Timirjazeva St., 16; tel.: (83952) 20-76-36; e-mail: iphr@sbamsr.irk.ru

Dolgikh Vladimir Valentinovich, PhD, Professor, Deputy Director of Scientific Center of Family Health and Human Reproduction Problems Siberian Branch of Russian Academy of Medical Sciences

Address: 664003, Irkutsk, Timirjazeva St., 16; tel.: (83952) 20-73-67; e-mail: iphr@sbamsr.irk.ru

Shoiko Sergei Valentinovich, PhD, Director of Territorial Fund of Mandatory Health Insurance of Citizens of Irkutsk Region Address: 664035, Irkutsk, July St., 3, 20; tel.: (83952) 34-19-02; e-mail: irotfoms@mccirk.ru

Abashin Nikolai Nikolaevich, PhD, Research Worker, the Laboratory of Public Health Problems, Scientific Center of Family Health and Human Reproduction Problems Siberian Branch of Russian Academy of Medical Sciences

Address: 664025, Irkutsk, Karl Marx St., 3; tel.: (83952) 24-03-34; e-mail: iphr@sbamsr.irk.ru

Lebedeva Lyudmila Nikolaevna, laboratory Assistant, Laboratory of Public Health Problems, Scientific Center of Family Health and Human Reproduction Problems Siberian Branch of Russian Academy of Medical Sciences

Address: 664025, Irkutsk, Karl Marx St., 3; tel.: (83952) 24-03-34; e-mail: leb_46@mail.ru 